\title{
Treating periodontal disease may improve metabolic control in diabetics
}

\author{
Abstracted from \\ Simpson TC, Needleman I, Wild SH, Moles DR, Mills EJ. \\ Treatment of periodontal disease for glycaemic control in people with diabetes. \\ Cochrane Database of Systematic Reviews 2010, Issue 5. \\ Address for correspondence: Luisa Fernandez Mauleffinch, Review Group Co-ordinator, \\ Cochrane Oral Health Group, MANDEC, School of Dentistry, University of Manchester, \\ Higher Cambridge Street, Manchester M15 6FH, UK. E-mail: luisa.fernandez@manchester.ac.uk
}

\section{Questions: Is there a relationship between periodontal therapy and glycaemic control in people with diabetes?}

Data sources The Cochrane Oral Health Group's Trials Register, The Cochrane Central Register of Controlled Trials (CENTRAL), Medline, EMBASE, CINAHL, ZETOC, ISIWeb of Knowledge and LILACS databases were searched together with hand searches of the journals Annals of Periodontology (1996 to 2003) and Periodontology 2000 (1993 to 2003). There were no language restrictions.

Study selection This review included randomised controlled trials of people with type 1 or 2 diabetes mellitus (DM) with a diagnosis of periodontitis. Suitable interventions included mechanical periodontal therapy with or without adjunctives and oral hygiene education. Data extraction and synthesis The titles and abstracts of all identified papers were examined by two review authors' independently. All included trials were assessed for risk of bias. Data were collated in evidence tables, grouped according to research design and assessed for possible meta-analysis on the basis of homogeneity of main characteristics.

Results Seven studies were included and three studies had results pooled into a meta-analysis. The effect for the mean percentage difference in $\mathrm{HbA} 1 \mathrm{c}$ for scaling/root planing and oral hygiene (+/- antibiotic therapy) versus no treatment/usual treatment after $3 / 4$ months was $-0.40 \%$ ( $95 \%$ confidence interval $(\mathrm{Cl})$ fixed effect $-0.78 \%$ to $-0.01 \%)$, representing a statistically significant reduction in $\mathrm{HbA1c}(\mathrm{P}=0.04)$ for scaling/root planing. One study was assessed as being at low risk of bias with the other two at moderate to high risk of bias. A subgroup analysis examined studies without adjunctive antibiotics $-0.80 \%$ (one study: $95 \% \mathrm{Cl}-1.73 \%$ to $0.13 \%$; $\mathrm{P}=0.09$ ), with adjunctive antibiotics in the test group $-0.36 \%$ (one study: $95 \%$ $\mathrm{Cl}-0.83 \%$ to $0.11 \% ; \mathrm{P}=0.14$ ), and with antibiotics in both test and control groups after $3 / 4$ months $-0.15 \%$ (one study: $95 \% \mathrm{Cl}-1.04 \%$ to $0.74 \% ; P=0.74$ ).

This paper is based on a Cochrane Review published in the Cochrane Library 2010, issue 5 (see www.thecochranelibrary.com for information). Cochrane Reviews are regularly updated as new evidence emerges and in response to feedback, and the Cochrane Library should be consulted for the most recent version of the review.
Conclusions There is some evidence of improvement in metabolic control in people with diabetes, after treating periodontal disease. There are few studies available and individually these lacked the power to detect a significant effect. Most of the participants in the study had poorly controlled type $2 \mathrm{DM}$ and there was little data from randomised trials on the effects on people with type $1 \mathrm{DM}$. Improving periodontal health is an important objective in itself. However, in order to understand the potential of this treatment to improve glycaemic control among people with diabetes, larger, carefully conducted and reported studies are needed.

\section{Commentary}

This is the first Cochrane review dealing with the effect of periodontal therapy on the activity/severity of a systemic disease. This work is without doubt the best available summary of the scientific literature addressing the effect of periodontal treatment in metabolic control in people with diabetes. Results are in favour of a significant effect of periodontal therapy in reducing HbA1c levels. Although still quite imprecise and apparently modest, this effect might translate into an important public health benefit, given the high prevalence of periodontitis on the one hand, and the mortality, morbidity and cost of diabetes on the other.

Other randomized controlled trials are still needed, and relevant implications for researchers are provided in this systematic review. Today, there is a broad consensus about the high quality of Cochrane systematic reviews, and the work by Simpson et al. fulfils all the quality requirements. This commentary will focus on some considerations that can help the general dental practitioner (GDP) to apply the results of this systematic review to his/her practice.

\section{Screening}

First, it is important for the GDP to ask new patients systematically about their diabetes status, and to cooperate actively with general practitioners and diabetologists. As stated by Simpson et al., "health providers may wish to explore ways of increasing co-operation between medical and dental/periodontal professionals involved in the care of people with diabetes". Then a full periodontal examination should be recommended for all known diabetic patients, as part of their routine oral health management. Indeed, people with diabetes experience periodontal disease more frequently and with greater severity than the general population. ${ }^{1,2}$ 
Information

Second, the GDP should give some information to diabetic patients with periodontitis:

- Achieving periodontal health is a worthwhile objective in relation to future oral health and wellbeing. Reported adverse effects of periodontal treatment among diabetic patients are quite similar to those observed in the general population: adverse effects related to chlorhexidine mouth rinses ${ }^{3}$, complications of antibiotic therapy and intraoral adverse effects. ${ }^{4}$

- Periodontal treatment could also be regarded as a possible way to improve glycaemic control. According to current scientific evidence, periodontal treatment (ie, scaling/root planing and oral health hygiene instructions) could lead to a mean percentage reduction of around $0.4 \%$ in HbA1c level. However, this value results from the meta-analysis of three studies, conducted on patients principally with type 2 diabetes, mainly aged 50 or over, and without additional major health problems.

- There is no evidence for a decrease in HbA1c values in diabetic patients who are different from those included in the present meta-analysis (eg, younger patients, patients with type 1 diabetes or multimorbidity). However, as improving periodontal health remains an important objective in itself, periodontal treatment should also be proposed to these patients.

\section{Treatment decision}

Third, the GDP must take the patient's characteristics into consideration and explain the risks and benefits of different treatment options (including no periodontal treatment). After consent, the GDPs should treat periodontitis according to their current practice and experience, the patient's characteristics and national guidelines (eg, regarding antibiotherapy, as the present systematic review is inconclusive as to the effect of adjunctive antibiotherapy). A regular periodontal maintenance schedule should also be proposed by the GDP.

In conclusion, it could be noted that other Cochrane systematic reviews on the effect of periodontal therapy on systemic diseases are in progress at present ${ }^{5-8}$ In the near future, they will allow GDPs to integrate high-quality, regularly updated, evidence-based information into their routine practice.

\section{Jean-Noel Vergnes \\ Department of Dentistry, Toulouse University Hospital (CHU de Toulouse) and Toulouse Dental School, Paul Sabatier University, Toulouse, France.}

1. Firlati $E$. The relationship between clinical periodontal status and insulin dependent diabetes mellitus. Results after 5 years. J Periodontol 1997; 68:136-140.

2. Sandberg 2000 Sandberg GE, Sundberg HE, Fjellstrom CA, Wikblad KF. Type 2 diabetes and oral health: a comparison between diabetic and nondiabetic subjects. Diabetes Res Clin Prac 2000; 50: 27-34.

3. McCoy LC, Wehler C], Rich SE, Garcia RI, Miller DR, Jones JA. Adverse events associated with chlorhexidine use: results from the Department of Veterans Affairs Dental Diabetes Study. J Am Dent Assoc 2008;139: 178-183.

4. Jones IA, Miller DR, Wehler Cl, Rich SE, Krall-Kaye EA, McCoy LC, et al. Does periodontal care improve glycemic control? The Department of Veterans Affairs Dental Diabetes Study. J Clin Periodontol 2007; 34: 46-52.

5. Crowther CA, Thomas N, Middleton P, Chua M, Esposito M. Treating periodontal disease for preventing preterm birth in pregnant women. (Protocol) Cochrane Database of Systematic Reviews 2005, Issue 2. Art. No.: CD005297. DOI: 10.1002/14651858.CD005297.

6. Vergnes et al. Interventions for periodontal disease in reducing the severity of rheumatoid arthritis. Registered Title, Cochrane Musculoskeletal Group, www2.cochrane.org/reviews/en/title C489741E82E26AA20113126E5EEB83A4.html

7. Yan X et al. Periodontal therapy as adjunctive treatment for gastric helicobacter pylori infection. Registered Title, Cochrane Upper Gastrointestinal and Pancreatic Diseases Group, www2.cochrane.org/reviews/en/title_206059996180216632541002081715 00.html

8. Li C et al. Periodontal therapy for the management of cardiovascular disease. Registered Title, Cochrane Oral Health Group, www2.cochrane.org/reviews/en/title 15375380671784407303100618181617.html

Evidence-Based Dentistry (2010) 11, 73-74. doi:10.1038/sj.ebd.6400734 